\title{
Suboptimal Birthweight and Adult Cardiometabolic Disease: The HPA-Axis Link
}

\author{
Wrivu N Martin ${ }^{1}$, Craig E Pennell ${ }^{1,2 *}$ and Carol Wang ${ }^{1,2}$ \\ ${ }^{1}$ School of Medicine and Public Health, University of Newcastle, Australia \\ ${ }^{2}$ Mothers and Babies Research Centre, Hunter Medical Research Institute, Australia
}

*Corresponding author: Craig E Pennell, School of Medicine and Public Health, Faculty of Medicine and Health, The University of Newcastle, Callaghan, New South Wales, Australia.

To Cite This Article: Wrivu N Martin, Craig E Pennell, Carol Wang, Suboptimal Birthweight and Adult Cardiometabolic Disease: The HPA-Axis Link. 2020 - 10(3). AJBSR.MS.ID.001520. DOI: 10.34297/AJBSR.2020.10.001520.

Received: 啙 September 21, 2020; Published: 畊 September 25, 2020

\section{Introduction}

Suboptimal birthweight $\left(<10^{\text {th }}\right.$ percentile, $>90^{\text {th }}$ percentile $)$ has a well-established relationship with several adult cardiometabolic risk factors, including obesity, dyslipidaemia and elevated blood pressure (BP) [1]. Over the past three decades, several animal studies have investigated this relationship. Together, these studies have shown that in sub-optimally grown offspring, adaptive changes occur in key organ systems to maximize chances of ex-utero survival [2]. Unfortunately however, these early life adaptations to organ structure and function render suboptimally grown offspring vulnerable to chronic disease later in life [3]. This concept is known as the Developmental Origins of Health and Disease (DoHAD) hypothesis, and provides the broad mechanistic framework underpinning the relationship between adverse early life exposures and chronic disease in adulthood [2]. The hypothalamic pituitary adrenal axis (HPA-A) plays a key role in cardiometabolic homeostasis by regulating tissue exposure to endogenous glucocorticoids [4]. Several animal studies have shown that the axis is highly susceptible to adaptive change in response to adverse early-life exposures [5].

Given that glucocorticoids induce gluconeogenesis, inhibit insulin secretion, enhance vascular responsiveness and promote lipid storage, growth driven HPA-A dysregulation may be an important mechanism through which suboptimal fetal growth is linked to increased cardiometabolic risk in adulthood. While independent relationships between these three components have been established $[1,6,7]$, the precise role of the HPA-A within this relationship remains unclear. As the global cardiometabolic phenotype continues to change [8], clarifying our understanding of the axis' role in facilitating cardiometabolic disease will become increasingly important.

\section{Hpa-Axis As A Mediator}

Several animal studies have suggested that suboptimal fetal growth may increase cardiometabolic risk by acting through changes to the HPA-A. For instance, nutritional deprivation (caloric restriction or low protein) among rat dams produces offspring with blunted HPA-A reactivity via alterations in hypothalamic glucocorticoid and mineralocorticoid receptor expression [9]. Similar outcomes have been observed in humans; in a metaanalysis of 2301 subjects from 11 separate studies, serum cortisol fell by $25.3 \mathrm{nmol} / \mathrm{L}$ for every kilogram increase in birthweight [6]. Although there is evidence that suboptimal fetal growth alters HPA-A function, whether this causally increases cardiometabolic risk remains unclear. There is some suggestion this might be the case; in pregnant guinea pigs, caloric restriction generates suboptimally grown offspring with increased basal and dynamic HPA-A activity, which is accompanied by elevated blood pressure and left ventricular hypertrophy [10].

Similarly, in a study of 370 men from the Hertfordshire cohort, birthweight was inversely related to serum cortisol levels (cortisol declined by $26.2 \mathrm{nmol} / \mathrm{L}$ for every $\mathrm{kg}$ increase in birthweight); intriguingly, higher cortisol levels were simultaneously associated with elevated blood pressure, fasting glucose, insulin resistance and serum triglycerides [11].

\section{HPA-Axis As A Moderator}

Genetics play an important role in HPA-A function, influencing glucocorticoid synthesis, metabolism and HPA-A responsiveness [12]. It is possible, therefore, that the relationship between suboptimal growth and elevated cardiometabolic risk is simply amplified among offspring who have inherited certain HPA-A 
genotypes, rather than suboptimal growth itself altering HPA-A function, and thereby producing cardiometabolic dysfunction. There are some important reasons why this may be the case. Firstly, genetic loci which are associated with low birth weight and elevated HPA-A function have been identified [13]. Failing to control for this crucial exposure-mediator confounder inflates the growth-HPA-A relationship, and thus falsely exaggerates the indirect pathway linking fetal growth, HPA-A function and cardiometabolic risk. More importantly however, given that excess fetal GC exposure is known to lower birth weight [14-16], suboptimal fetal growth may simply be a manifestation of elevated maternal HPA-A function which the fetus inherits; this inheritance then ultimately increases their cardiometabolic risk [7].

This concept was recently highlighted in a large Mendelian randomization study which demonstrated that higher blood pressure among offspring of low birth weight is best explained by their inheritance of maternal alleles known to elevate blood pressure [17]. Indeed, among this cohort, low birth weight was simply a manifestation of the intrauterine consequences of elevated maternal BP. Alternatively, increased HPA-A function and suboptimal fetal growth may simply interact, irrespective of their origins, to produce elevated cardiometabolic risk. We recently performed an analysis supporting this possibility in 851 young adults, where we found that the HPA-A is not a mediator of the relationship between birthweight and cardiometabolic outcomes [18]. Rather, our analyses showed that this relationship varied by HPA-A phenotype. In our study, we observed elevated blood pressure among participants of low birthweight with anticipatory HPA-A profiles, while excessively grown offspring with nonresponsive HPA-A profiles had higher BMI. Further genetic studies among additional cohorts will be needed to confirm these findings.

\section{Future Directions}

As the global burden of cardiometabolic disease expands, novel approaches are needed to identify vulnerable individuals at a population level. Although suboptimal fetal growth has been identified as an important modifiable risk factor, its mechanisms remain elusive. The HPA-A plays an important role in cardiometabolic homeostasis. As we continue to dissect its role in the growth-risk relationship, well-powered studies incorporating life-course data will be necessary. Assumptions about intrauterine programming must also evolve as new evidence emerges, along with our approaches to establishing causality.

\section{References}

1. Knop MR, Geng TT, Gorny AW (2018) Birth Weight and Risk of Type 2 Diabetes Mellitus, Cardiovascular Disease, and Hypertension in Adults: A Meta-Analysis of 7646267 Participants from 135 Studies. Journal of the American Heart Association 7(23): e008870.

2. Gluckman PD, Hanson MA, Buklijas T (2010) A conceptual framework for the developmental origins of health and disease. Journal of developmental origins of health and disease 1(1): 6-18.
3. Hanson MA, Gluckman PD (2014) Early developmental conditioning of later health and disease: physiology or pathophysiology? Physiological reviews 94(4): 1027-1076.

4. Moisiadis VG, Matthews SG (2014) Glucocorticoids and fetal programming part 1: Outcomes. Nature reviews Endocrinology 10(7): 391-402.

5. Moisiadis VG, Matthews SG (2014) Glucocorticoids and fetal programming part 2: Mechanisms. Nature reviews Endocrinology 10(7): 403-411.

6. van Montfoort N, Finken Mj Fau le Cessie S, le Cessie S Fau Dekker FW (2005) could cortisol explain the association between birth weight and cardiovascular disease in later life? A meta-analysis. Eur J Endocrinal 153(6): 811-817.

7. Crawford AA, Soderberg S, Kirschbaum C(2019)Morning plasma cortisol as a cardiovascular risk factor: findings from prospective cohort and Mendelian randomization studies. Eur J Endocrinal 181(4): 429-438.

8. Bennett JE, Stevens GA, Mathers CD (2018) NCD Countdown 2030: worldwide trends in non-communicable disease mortality and progress towards Sustainable Development Goal target 3.4. The Lancet 392(10152): 1072-1088.

9. Yam KY, Naninck EF, Schmidt MV (2015) Early-life adversity programs emotional functions and the neuroendocrine stress system: the contribution of nutrition, metabolic hormones and epigenetic mechanisms. Stress (Amsterdam, Netherlands) 18(3): 328-342.

10. Bertram C, Khan O, Ohri S (2008) Transgenerational effects of prenatal nutrient restriction on cardiovascular and hypothalamic-pituitaryadrenal function. The Journal of physiology 586(8): 2217-2229.

11. Phillips DI, Barker DJ, Fall CH (1998) Elevated plasma cortisol concentrations: a link between low birth weight and the insulin resistance syndrome? J Clin Endocrinol Metab 83(3): 757-760.

12. Gerritsen L, Milaneschi Y, Vinkers CH (2017) HPA Axis Genes, and Their Interaction with Childhood Maltreatment, are related to Cortisol Levels and Stress-Related Phenotypes. Neuropsychopharmacology 42(12): 2446-2455.

13. Anderson LN, Briollais L, Atkinson HC(2014) Investigation of genetic variants, birthweight and hypothalamic-pituitary-adrenal axis function suggests a genetic variant in the SERPINA6 gene is associated with corticosteroid binding globulin in the western Australia pregnancy cohort (Raine) study. PloS one 9(4): e92957.

14. Reynolds RM (2012) Nick Hales Award Lecture 2011: glucocorticoids and early life programming of cardiometabolic disease. Journal of developmental origins of health and disease 3(5): 309-314.

15. Reynolds RM (2013) Glucocorticoid excess and the developmental origins of disease: two decades of testing the hypothesis--2012 Curt Richter Award Winner. Psychoneuroendocrinology 38(1): 1-11.

16. Duthie L, Reynolds RM (2013) Changes in the Maternal HypothalamicPituitary-Adrenal Axis in Pregnancy and Postpartum: Influences on Maternal and Fetal Outcomes. Neuroendocrinology 98(2): 106-115.

17. Warrington NM, Beaumont RN, Horikoshi M (2019) Maternal and fetal genetic effects on birth weight and their relevance to cardio-metabolic risk factors. Nature genetics 51(5): 804-814

18. Martin WN (2019) early life programming of the hypothalamic-pituitaryadrenal axis: does it mediate the relationship between fetal growth and adult cardiometabolic outcomes? 